\title{
Comparative Myocardial Deformation in 3 Myocardial Layers in Mice by Speckle Tracking Echocardiography
}

\author{
Nicole Tee, ${ }^{1}$ Yacui Gu, ${ }^{1}$ Murni, ${ }^{1}$ and Winston Shim ${ }^{1,2}$ \\ ${ }^{1}$ National Heart Research Institute Singapore, National Heart Centre Singapore, Singapore 169609 \\ ${ }^{2}$ Duke-NUS, Graduate Medical School, Singapore 169857
}

Correspondence should be addressed to Winston Shim; winston.shim.s.n@nhcs.com.sg

Received 6 October 2014; Accepted 18 January 2015

Academic Editor: James Kirkpatrick

Copyright (C) 2015 Nicole Tee et al. This is an open access article distributed under the Creative Commons Attribution License, which permits unrestricted use, distribution, and reproduction in any medium, provided the original work is properly cited.

\begin{abstract}
Background. Speckle tracking echocardiography (STE) using dedicated high-resolution ultrasound is a relatively new technique that is useful in assessing myocardial deformation in 3 myocardial layers in small animals. However, comparative studies of STE parameters acquired from murine are limited. Methods. A high-resolution rodent ultrasound machine (VSI Vevo 2100) and a clinically validated ultrasound machine (GE Vivid 7) were used to consecutively acquire echocardiography images from standardized parasternal long axis and short axis at midpapillary muscle level from $13 \mathrm{BALB} / \mathrm{c}$ mice. Speckle tracking strain (longitudinal, circumferential, and radial) from endocardial, myocardial, and epicardial layers was analyzed using vendor-specific offline analysis software. Results. Intersystem differences were not statistically significant in the global peak longitudinal strain $(-16.8 \pm 1.7 \%$ versus $-18.7 \pm 3.1 \%)$ and radial strain $(46.8 \pm 14.2 \%$ versus $41.0 \pm 9.5 \%)$, except in the global peak circumferential strain $(-16.9 \pm 3.1 \%$ versus $27.0 \pm 5.2 \%, P<0.05)$. This was corroborated by Bland Altman analysis that revealed a weak agreement in circumferential strain (mean bias \pm 1.96 SD of $-10.12 \pm 6.06 \%$ ) between endocardium and midmyocardium. However, a good agreement was observed in longitudinal strain between midmyocardium/endocardium (mean bias $\pm 1.96 \mathrm{SD}$ of $-1.88 \pm 3.93 \%$ ) and between midmyocardium/epicardium (mean bias \pm 1.96 SD of $3.63 \pm 3.91 \%$ ). Radial strain (mean bias \pm 1.96 SD of $-5.84 \pm 17.70 \%$ ) had wide limits of agreement between the two systems that indicated an increased variability. Conclusions. Our study shows that there is good reproducibility and agreement in longitudinal deformation of the 3 myocardial layers between the two ultrasound systems. Directional deformation gradients at endocardium, myocardium, and epicardium observed in mice were consistent to those reported in human subjects, thus attesting the clinical relevance of STE findings in murine cardiovascular disease models.
\end{abstract}

\section{Introduction}

Two-dimensional (2D) speckle tracking echocardiography (STE) has improved quantification of wall motion deformation in assessing cardiac performance. The STE technique captures myocardial features in greyscale $B$-mode images from interference of reflected ultrasound beam and presents them as unique speckle patterns [1]. Postprocessing of the speckle patterns through user-defined region of interest in image pixels and tracking their movement enable extraction of spatial and temporal data. These yield useful regional velocity, displacement, strain, and strain rate along the longitudinal, radial, and circumferential axes of the left ventricle. The STE technique has an advantage over tissue Doppler imaging (TDI) in angle independent assessments [2] and it is known to be highly reproducible when compared to 2D TDI and 3D TDI in clinical imaging [3].

STE is gaining clinical importance due to compelling validation against data gathered from magnetic resonance imaging (MRI), TDI, and sonomicrometry techniques in animal models [4-7] and in clinical settings [8-10]. However, it is recognized that different vendors employed disparate speckle tracking algorithms that are largely proprietary and comparative studies of different STE systems have not been extensively reviewed $[11,12]$. Efforts by the American Society of Echocardiography (ASE) and the European Association of Echocardiography (EAE) to standardize analytical software have had limited success [1]. The usefulness of STE in 
identifying segmental LV dysfunction in mouse heart failure model has been demonstrated previously using a conventional clinical echocardiography system [13] and a highresolution rodent ultrasound system [14]. However, crosscomparability of data acquired by the two ultrasound systems is unclear. Therefore, we sought to examine data consistency of $2 \mathrm{D}$ speckle-derived myocardial strain data captured and analyzed by a dedicated rodent Vevo 2100 system with a clinically validated Vivid 7 system to verify clinical relevance of our experimental findings in healthy mice.

\section{Methods}

2.1. Animal Preparation. All animal studies conducted were approved by Institutional Animal Care and Use Committees. A total of 13 male BALB/c mice were used. Mice were anesthetized at $2 \%$ isoflurane with $1 \mathrm{~L} / \mathrm{hr}$ oxygen during induction for 20 minutes and were maintained at $1 \%$ to $1.5 \%$ isoflurane during imaging. Mice were fixed in the supine position on a heated platform with paws secured to the electrode pads covered with conducting gel for ECG monitoring when scanning with Vevo 2100 (VisualSonics, VSI, Toronto, Canada) system. ECG electrodes were placed onto the left and right limbs and left upper extremity of the mice when scanning with GE Vivid 7 (GE Healthcare, Horten, Norway). All heart rates (HR) were monitored and maintained at the average of 360-460 bpm. Hair removal cream was applied onto the chest, neckline, and upper and lower extremities of the mice. Care was taken to avoid excessive pressure while acquiring images, which was known to induce bradycardia.

2.2. Echocardiographic Image Acquisition. Echocardiography was performed on GE Vivid 7 with i13L linear array transducer and Vevo 2100 with MS400 linear array transducer (Table 1). To ensure reproducibility, segments or images with acoustic shadowing or reverberations were omitted from the study. Special care was taken to optimize sector width for complete myocardial visualization while artifacts that resemble speckles influencing tracking quality were precluded. Gain settings were adjusted to optimize endocardial definition. Extra care was taken to maintain high frame rate to circumvent shifting of speckles in sequential frames without compromising imaging quality associated with reduced number of ultrasound beams in each frame in sustaining high frame rates [15]. Standard parasternal long axis (Figure 1) and short axis at midpapillary muscle level (Figure 2) views with frame rate more than 200 frames/sec with Vevo 2100 were obtained as per vendor recommendation for optimal speckle tracking analysis. Frame rate of 130190 frames/sec was obtained with Vivid 7. Foreshortening view was omitted as it tended to underestimate true strain, thus affecting 2D STE results [15]. 2D guided $M$-mode of parasternal short axis at papillary muscle level was acquired to measure LV conventional parameters. Average of 10 cardiac cycles at each plane was stored in cineloop with both systems for subsequent offline analysis.
TABLE 1: Comparison between GE Vivid 7 and Vevo 2100 in 2Dspeckle tracking hardware and software abilities.

\begin{tabular}{lcc}
\hline & Hardware abilities & \\
& GE Vivid 7 & Vevo 2100 \\
\hline Transducer frequency & $10-14 \mathrm{MHz}$ & $18-38 \mathrm{MHz}$ \\
Axial resolution & $200 \mu \mathrm{m}$ & $50 \mu \mathrm{m}$ \\
Lateral resolution & $300 \mu \mathrm{m}$ & $110 \mu \mathrm{m}$ \\
Transducer footprint & $28 \mathrm{~mm} \times 10 \mathrm{~mm}$ & $20 \mathrm{~mm} \times 5 \mathrm{~mm}$ \\
Temporal resolution & $130-190 \mathrm{fps}$ & $>200 \mathrm{fps}$ \\
Detect respiratory cycle & Yes & Yes \\
Image acquisition & Conventional & $\begin{array}{c}\text { Hand-free }+ \\
\text { conventional }\end{array}$ \\
Area of analysis & Myocardium & Endocardium + \\
epicardium \\
ROI adjustment & Uniform & Individual \\
Ability to distinguish E/A & & Yes \\
wave when heart rate is more & No & No \\
than 400 bpm & & Yutomated \\
Marked AVC & Manual & \\
FAC & &
\end{tabular}

fps: frame per second, AVC: aortic valve closure, and FAC: fractional area change (defined as cross-sectional area change between end diastole and end systole).

2.3. Postprocessing Analysis. 2D STE applied myocardial lagrangian strain by following movement of stable patterns of acoustic markers frame by frame throughout the cardiac cycle. The shift of these acoustic markers represented tissue movement and provided spatial and temporal data used to calculate changes in length of the myocardium with the use of vendor-specific analysis software. Global peak radial (RS) and circumferential strains (CS) sampled from anterior, lateral, posterior, inferior, posteroseptal, and anteroseptal segments were measured from the short axis view. Clinically, global peak longitudinal strain (LS) is measured from apical 4chamber view; however due to the anatomical position of rodent heart, apical 4-chamber view was not feasible; instead global peak LS was measured from anterior basal, mid, and apical and posterior basal, mid, and apical segments of long axis view. Midmyocardium strain data of GE images were analyzed by 2D-strain EchoPAC PC version 103.0.1 (GE Healthcare, Horten, Norway) while strain data from epicardial and endocardial segments of Vevo images were analyzed by VevoStrain version 1.3.0. (Visual Sonics, VSI, Toronto, Canada). The endocardial border was manually traced at the end systolic frame by point and click approach. Epicardial border was assimilated by the software automatically and was verified and accepted for analysis when no further adjustments were required. Segments with inadequate tracing were excluded from analysis.

2D guided $M$-mode of parasternal short axis was used to measure end diastolic diameter (LVEDD) and end systolic diameter (LVESD). Ejection fraction was calculated as $\operatorname{LVEF}(\%)=\left[\left(\operatorname{LVEDD}-\mathrm{LVESD}^{2} / \mathrm{LVEDD}^{2}\right] \times 100\right.$. Fractional shortening was calculated as FS (\%) $=[($ LVEDD LVESD)/LVEDD] $\times 100$. All image acquisitions and offline 


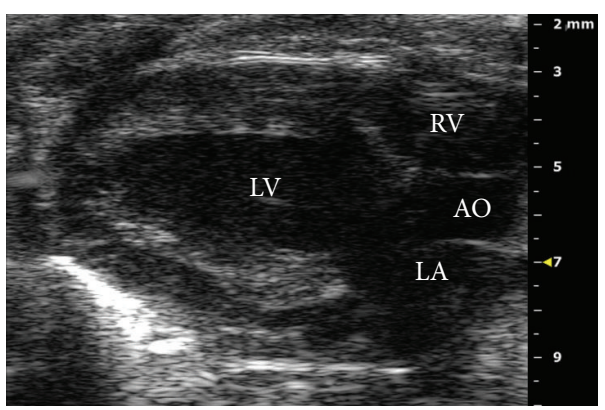

(a)

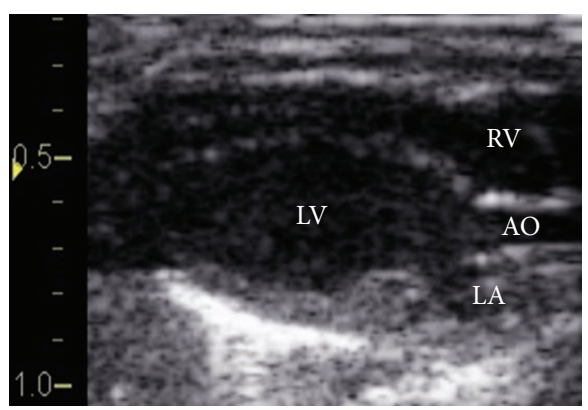

(b)

FIGURE 1: 2D greyscale of parasternal long axis acquired by (a) VSI Vevo 2100 and (b) GE Vivid 7. LV: left ventricle; RV: right ventricle; LA: left atrium; AO: aorta.

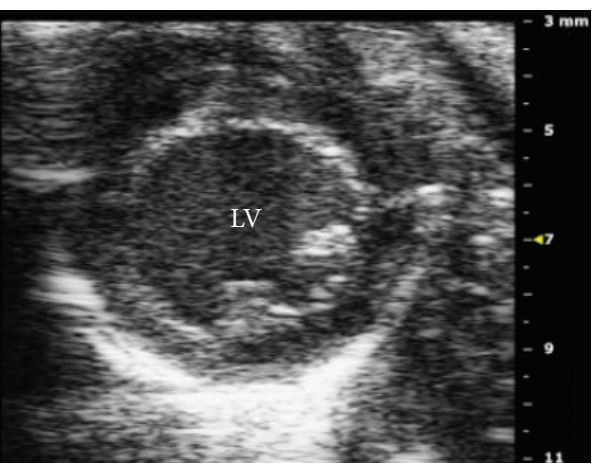

(a)

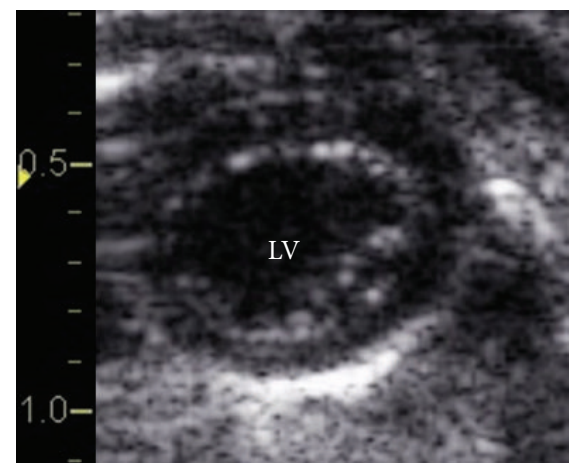

(b)

FIGURE 2: 2D greyscale of parasternal short axis acquired by (a) VSI Vevo 2100 and (b) GE Vivid 7. LV: left ventricle.

measurements were conducted by an experienced sonographer. Unlike EchoPAC, VevoStrain does not display aortic valve closure (AVC) and is not as apparent in detecting delay in LV contraction. However, the AVC displayed on strain measurement is based on the AVC marked down in left ventricle outflow tract (LVOT) pulse wave Doppler in EchoPAC. Therefore, heart rate during the Doppler analysis might differ during the strain analysis. In such case, the AVC timing has to be corrected by heart rate through the following formula: $\mathrm{AVC}_{s}=\mathrm{AVC}_{d} \times\left(R-R_{s} / R-R_{d}\right)^{1 / 2}$, where $R-R_{s}$ interval derived from $2 \mathrm{D}$ strain and $R-R_{d}$ and $\mathrm{AVC}_{d}$ derived from Doppler tracing. $\mathrm{AVC}_{s}$ is aortic valve closure time in $2 \mathrm{D}$ strain.

2.4. Statistical Analysis. Data were presented as mean \pm standard deviation. Paired $t$-test was used to detect any significant difference in conventional echo parameters and strain measurements. $P$ values $<0.05$ were considered statistically significant. Global peak strain was calculated as the average of all measurable segments. The mean difference and limits of agreement ( $95 \%$ confidence interval) between measurements derived from each system were calculated. Agreement between systems was determined by Bland Altman analysis [16]. Five randomly selected mice were reanalyzed for their global peaks LS, CS, RS, and LVEF by a second sonographer to determine interobserver variability. Interobserver agreement was calculated using intraclass correlation coefficient [17]. Statistical analysis was performed using SPSS software (version 20.0, SPSS Inc., Chicago, IL, USA).

\section{Results}

A total of 78 segments were acquired (6 segments each view $\times 13$ animals). Midmyocardium LS (-16.8 $\pm 1.7 \% ; 74 / 78$ segments analyzed) by EchoPAC was not statistically different as compared to endocardial LS $(-18.7 \pm 3.1 \%, P=0.11,76 / 78$ segments analyzed), but it was significantly different from those in epicardial LS $(-13.2 \pm 4.3 \%, P<0.05,70 / 78$ segments analyzed) by VevoStrain (Table 3 ).

Midmyocardium CS (-16.9 $\pm 3.1 \%, 74 / 78$ segments analysed) by EchoPAC differed significantly from endocardial CS $(-27.0 \pm 5.2 \%, P<0.05,77 / 78$ segments analyzed $)$ and epicardial CS $(-11.3 \pm 2.0 \%, P<0.05,76 / 78$ segments analyzed) measured using VevoStrain (Table 3). Peak RS did not differ significantly between EchoPAC (46.8 $\pm 14.2 \%$, $P=0.26,75 / 78$ segments analysed) and VevoStrain (41.0 \pm 9.5\%, 76/78 segments analyzed) (Table 3). No significant differences were found in mean HR computed by EchoPAC or VevoStrain $(P=0.245)$. Similarly, $\operatorname{LVEF}(P=0.13)$ and FS $(P=0.11)$ from $M$-mode analysis were not significantly different between the two systems (Table 2). 
TABLE 2: Consecutively acquired GE Vivid 7 and Vevo 2100 conventional parameters.

\begin{tabular}{lcccc}
\hline & GE & VSI & P value & 95\% CI \\
& EchoPAC & VevoStrain & & \\
\hline Mean HR (bpm) & $401 \pm 82$ & $384 \pm 46$ & NS & -67.2 to 33.2 \\
Mean LVEF (\%) & $59.6 \pm 7.5$ & $57.6 \pm 7.3$ & NS & -0.27 to 4.2 \\
Mean FS (\%) & $36.7 \pm 6.1$ & $35.1 \pm 5.9$ & NS & -0.11 to 3.3 \\
\hline
\end{tabular}

Analysis by scatter diagrams and Bland Altman plots showed that global peak LS had a better agreement than global peak CS and RS between the two systems (Figure 3). The mean bias and limits of agreement (1.96 SD) between endocardial LS and midmyocardium LS was smallest at $-1.88 \pm 3.93 \%$ and followed by between epicardial LS and midmyocardium LS at $3.63 \pm 3.91 \%$. The peak CS was identified to have a major bias with mean bias $\pm 1.96 \mathrm{SD}$ of $-10.12 \pm 6.06 \%$ and $5.57 \pm 3.41 \%$ at endocardial and epicardial segments, respectively. Mean bias in peak RS was found to have the widest limits of agreement with mean difference of $-5.84 \pm 17.70 \%$ (Figure 3 ).

The variability of global peaks LS, CS, and RS measurements using EchoPAC between two independent observers was highly reproducible with intraclass correlation coefficient at $1.0,0.79$, and 0.94 , respectively. The variability of global peak LS for endocardium and epicardium analyzed using VevoStrain showed good correlation at 0.97 and poorer one at 0.55 , respectively. While variability of global peak CS for endocardium and epicardium was 0.93 and 0.54 , respectively, variability of global peak RS measured was 0.93 .

\section{Discussion}

Mouse represents a critical model in the understanding of LV dysfunction in cardiovascular diseases. Changes to LV structure and function detected by conventional echocardiographic parameters, such as fractional shortening (FS) or ejection fraction (EF), are considered to be late manifestation of disease. In contrast, STE and speckle tracking-based strain analysis are found to provide greater sensitivity and specificity in detecting subtle early changes of cardiac performance in cardiac pathophysiology.

There are increasing compelling data validations of $2 \mathrm{D}$ STE against data from MRI, tissue doppler imaging, and sonomicrometry in animal models and clinical studies in detecting abnormal LV function are emerging $[4,5]$. Nevertheless, considerable challenges remain in STE imaging of rodents due to their small size, heart orientation, and rapid heart rates. High-resolution rodent ultrasound systems have been introduced to circumvent such limitations in STE and strain analysis $[6,14,18]$. It is assumed that strain calculation of the relative change in length between individual speckle by formula $(\varepsilon)=\left(L_{1}-L_{0}\right) / L_{0}$, whereby $L_{0}$ is the original length. However, it is recognized that different systems employed disparate speckle tracking algorithms that are largely proprietary with unknown cross comparability. Comparative data between different STE systems have not been extensively reported $[11,12]$.
We observed in this study that there was a better agreement of LS measurements between the two systems than the CS and RS measurements by Bland Altman plots. Furthermore, better agreement was shown in longitudinal strain between midmyocardium and endocardium (mean bias of $-1.88 \%$; $1.96 \mathrm{SD}$ of $\pm 3.93 \%$ ) than with epicardium (mean bias of $3.63 \%$; $1.96 \mathrm{SD}$ of $\pm 3.91 \%$ ). This coincided with clinically observed parallel gradient for longitudinal strain between endocardial and midmyocardial layers, but not epicardium and midmyocardium [19], which supported the layer-specific statistical differences observed in strain values (Table 3). The weak agreement observed $(-10.12 \%)$ in the Bland Altman plot of circumferential strain between endocardium and midmyocardium may be accentuated by differential muscle fiber orientation between the midmyocardial layer (mainly circumferential) and its two adjacent layers (mainly longitudinal) that affects myocardial layer deformation characteristics [12].

It is well recognized that LS and CS are the highest in endocardium followed by myocardium and epicardium in healthy human subjects, though discrepancy has also been noted $[19,20]$. Consistently, our study in mice similarly found that VevoStrain's endocardial LS produced the highest value $(-18.7 \pm 3.1 \%)$, and epicardial LS recorded the lowest value $(-13.2 \pm 4.3 \%)$ while EchoPAC's midmyocardium LS reported an intermediate value $(-16.8 \pm 1.7 \%)$ that is in agreement with the "averaging effect" in EchoPAC, where values generated represent the mean of all three cardiac layers [12]. Similar concordance results on CS values were recorded, whereby VevoStrain endocardial strain reported a higher CS value $(27.0 \pm 5.2 \%)$ than that of EchoPAC's midmyocardial strain $(-16.9 \pm 3.1 \%)$, while epicardial strain displayed the lowest CS value $(11.3 \pm 2.0 \%)$. These findings reaffirmed the clinical relevance of STE from preclinical experimental models.

Due to different design of the two transducers (Figure 4), i13L of the GE system offers a more effortless and flexible imaging, though the MS400 of Vevo 2100 affords an additional option of hand-free handling. Similar to percentage excluded segments reported previously [12], about $11 \%$ of the excluded LS segments analyzed using Vevo 2100 were basal anterior segment where the images were obscured or affected by shadows from sternum (Figure 5(a)). We minimized such obstruction by adjusting transducer angle, but at the expense of tilting of left ventricle apex (Figure 5(b)). However, this did not affect data integrity as STE has the advantage of angle independence as compared to TDI. The Vevo 2100 machine provides animal handling and physiological monitoring system that tracks not only heart rate more than 300 beats per minute, but also animal's respiratory cycle and temperature. We found it most useful in choosing good cardiac cycle during expiration of the respiratory cycle for analysis as cardiac strain analysis during inspiration invariably produced poorer results.

Radial strain (RS) values from VevoStrain were derived from taking corresponding points at the epicardium and endocardium and averaging them across the radial distance between the two points (Figure 6). Our study did not reveal significant RS differences between our two ultrasound systems. However, we found that RS has a wide limit of 


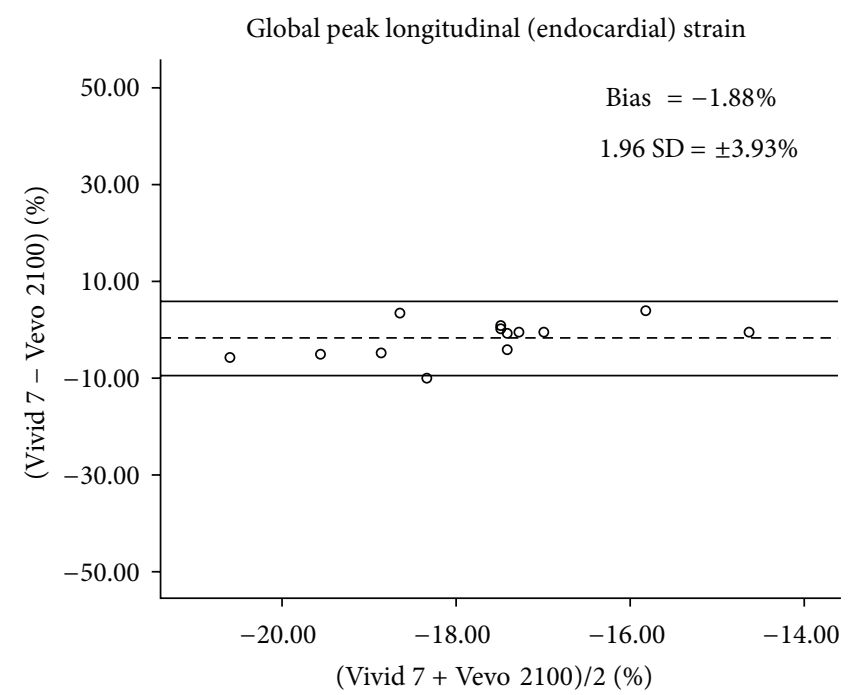

(a)

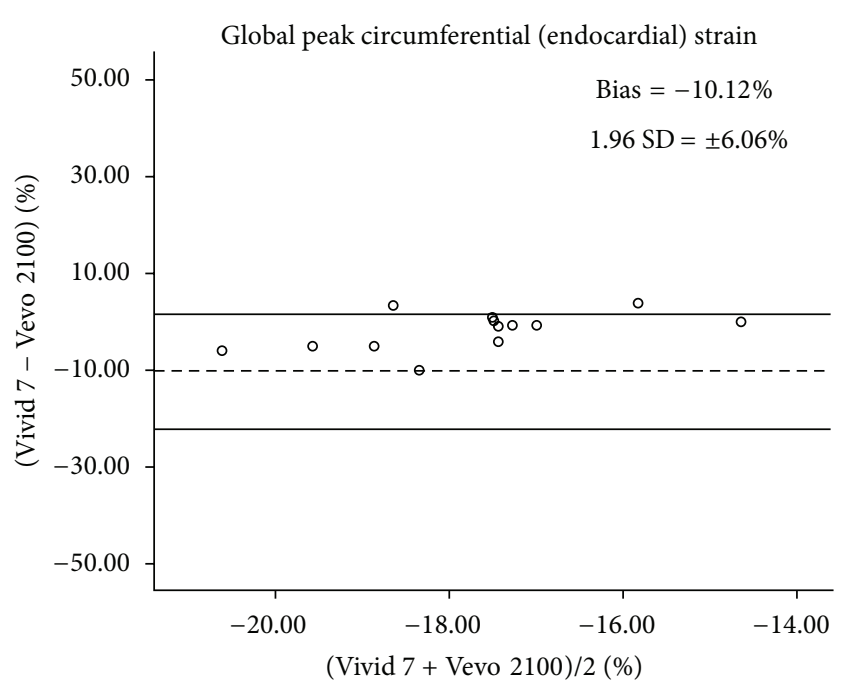

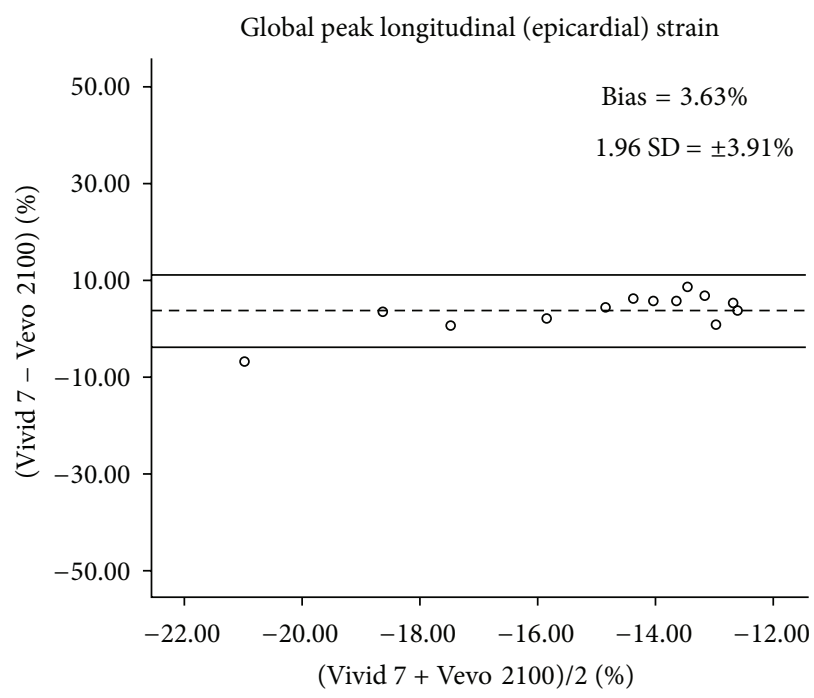

a)

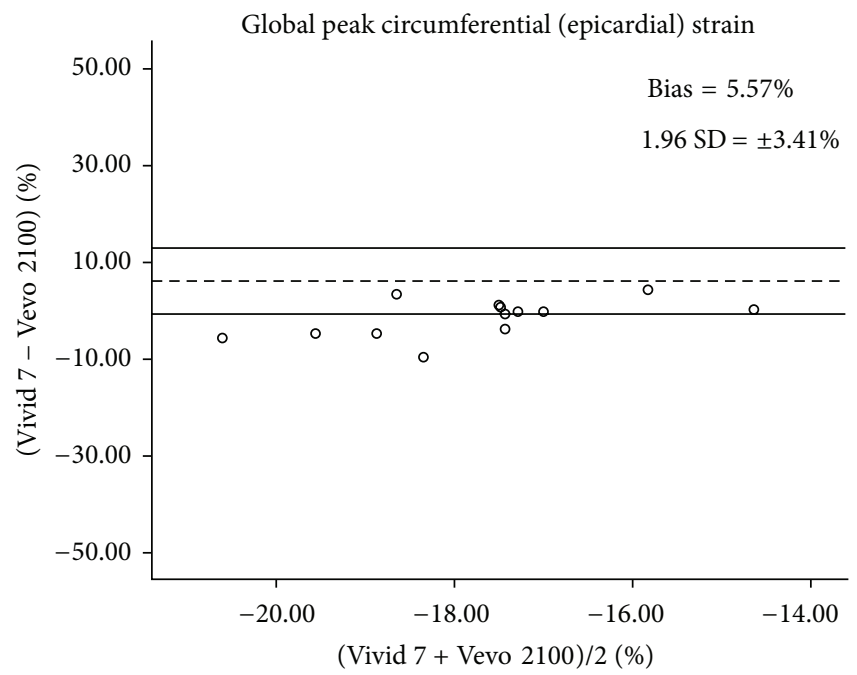

(b)

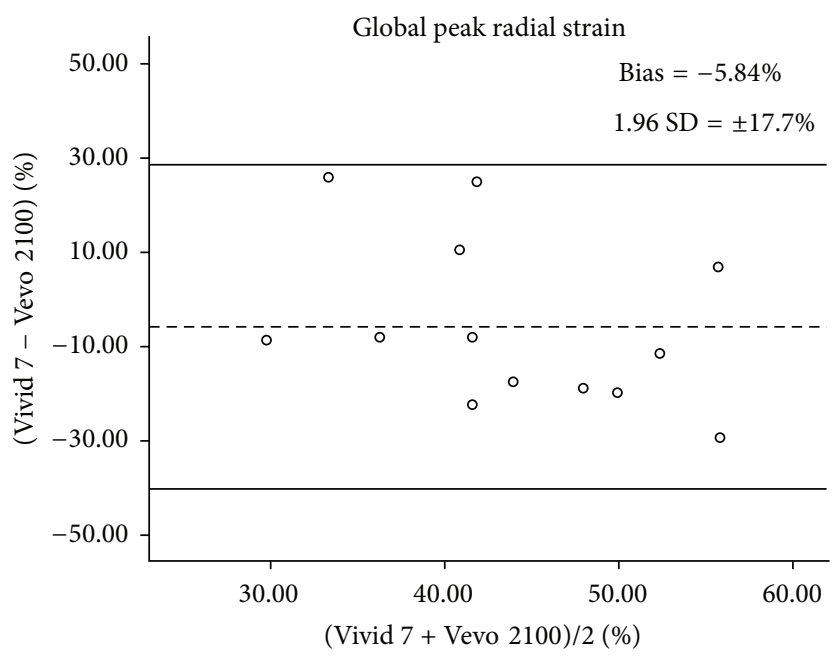

(c)

FIGURE 3: Bland Altman plot depicts the agreement of strain analysis between EchoPAC (Vivid 7) and VevoStrain (Vevo 2100). (a) Global peak longitudinal strain between midmyocardium/endocardium and midmyocardium/epicardium shows narrower variation than (b) global peak circumferential strain and (c) global peak radial strain. Dotted horizontal lines denote bias (mean difference between two systems) and solid horizontal lines illustrate the $95 \%$ limits of agreement. 
TABLE 3: Consecutively acquired GE Vivid 7 and Vevo 2100 global peak strains.

\begin{tabular}{lcccc}
\hline & GE EchoPAC & VSI VevoStrain & $P$ value & $95 \%$ CI \\
\hline Global peak longitudinal strain (\%) & $-16.8 \pm 1.7$ & $-18.7 \pm 3.1$ (Endo) & NS & -4.2 to 0.5 \\
& & $-13.2 \pm 4.3$ (Epi) & 0.006 & 1.3 to 6.0 \\
\hline Global peak circumferential strain (\%) & $-16.9 \pm 3.1$ & $-27.0 \pm 5.2$ (Endo) & $<0.05$ & -13.8 to -6.5 \\
& & $-11.3 \pm 2.0$ (Epi) & $<0.05$ & 3.5 to 7.6 \\
\hline Global peak radial strain (\%) & $46.8 \pm 14.2$ & $41.0 \pm 9.5$ & NS & -16.5 to 4.9 \\
\hline
\end{tabular}

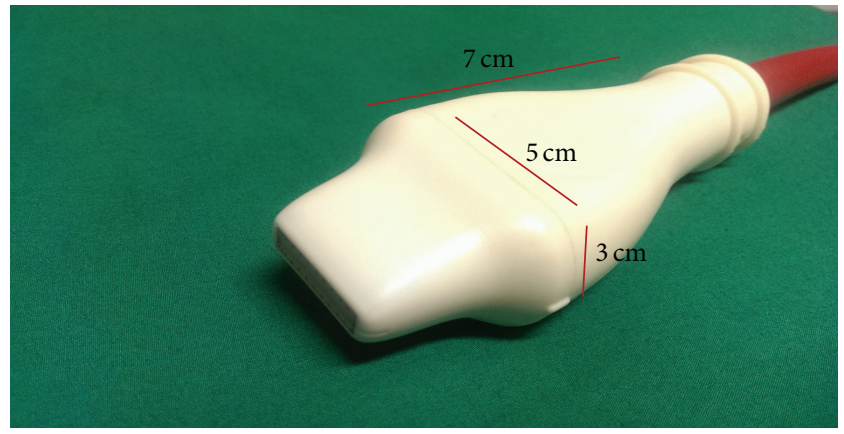

(a)

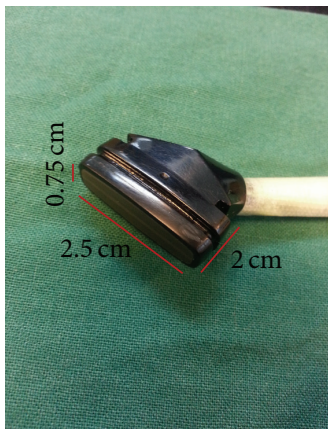

(b)

FIGURE 4: VSI MS 400 transducer (a) with frequency of 18-38 MHz. GE i13L transducer (b) with frequency of 10-14 MHz.

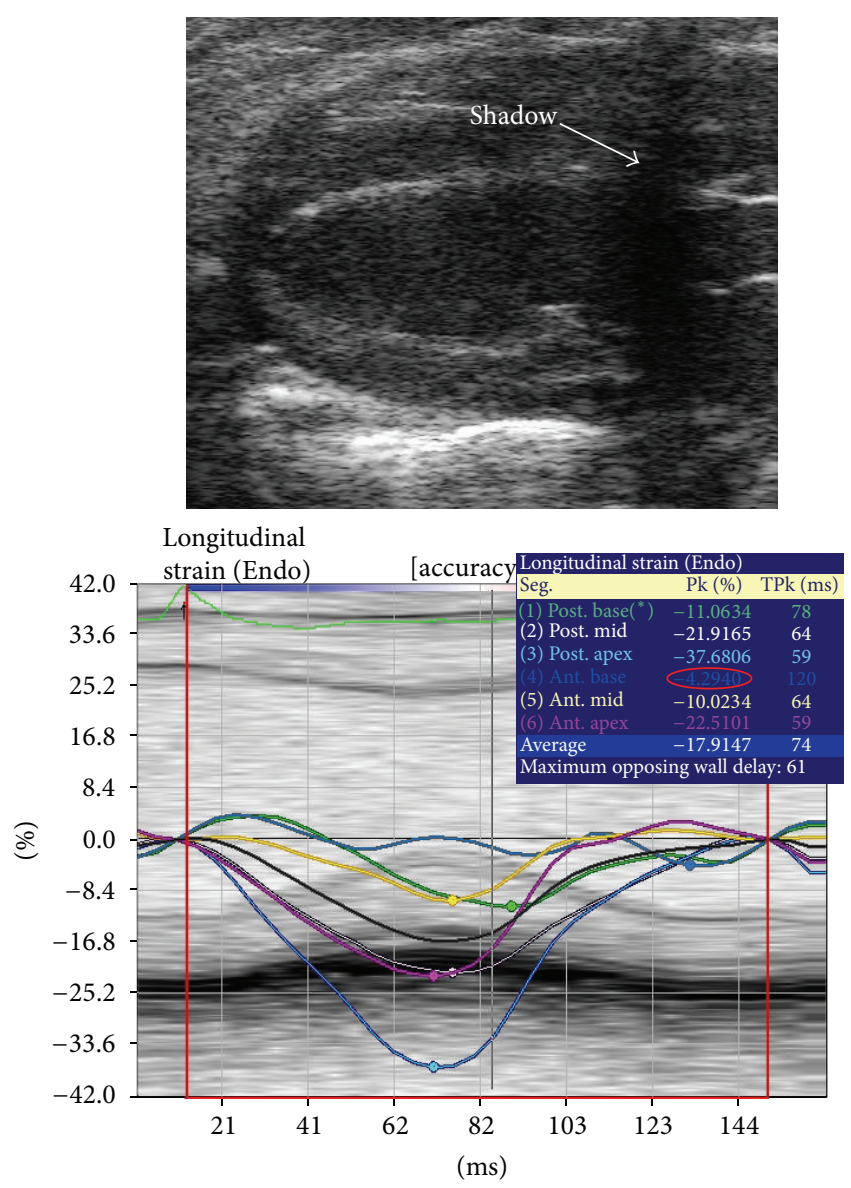

(a)
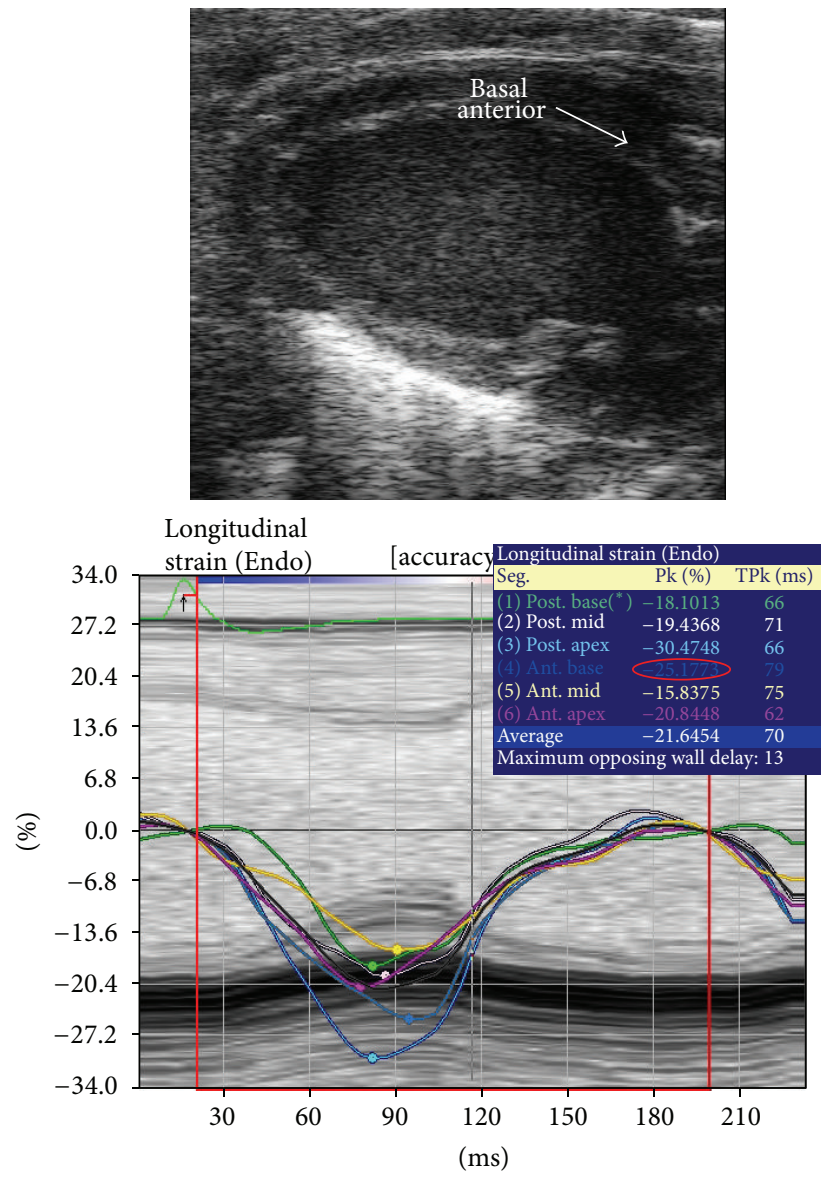

(b)

FIGURE 5: Representation of 2D parasternal long axis where (a) showed basal anterior obscured by shadowing due to sternum but with slight adjustment; shadowing can be eliminated (b). Speckle tracking (below) showed a significant difference in the reading. 


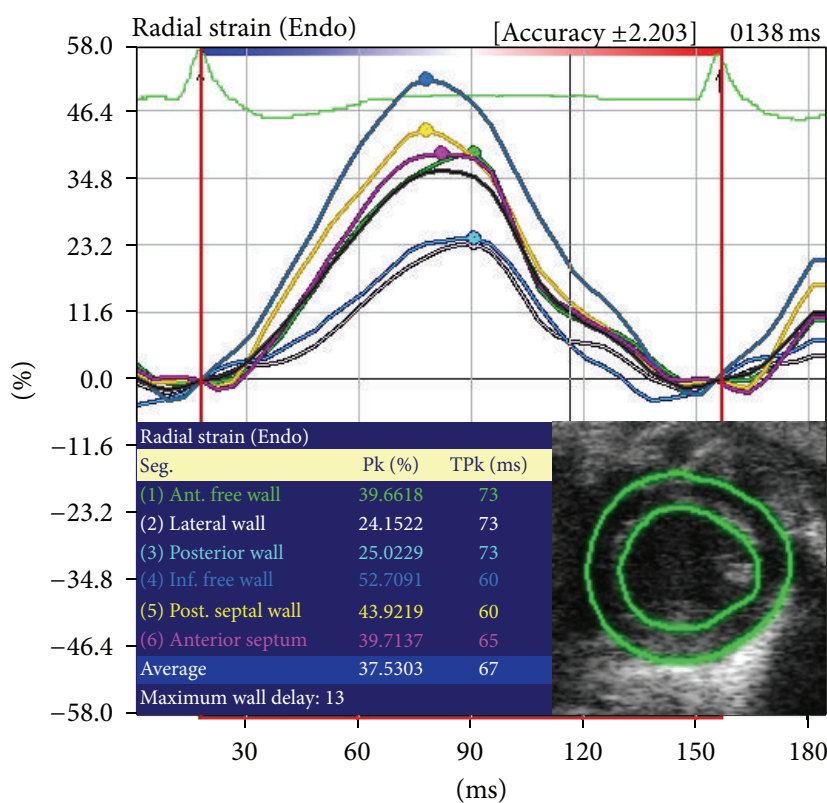

(a)

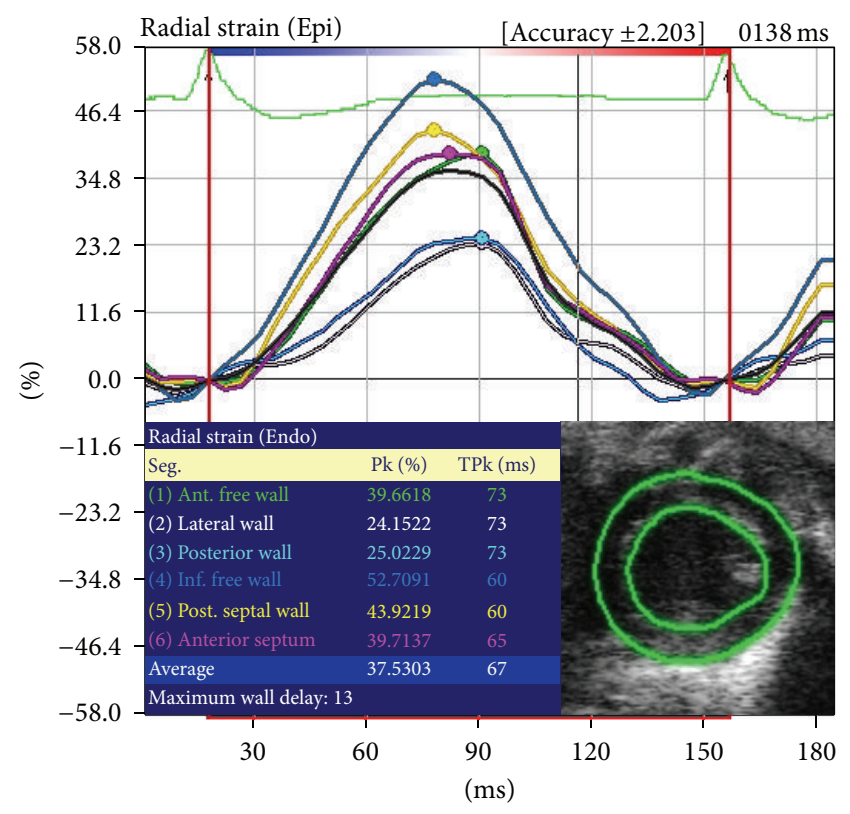

(b)

FIGURE 6: Representation of VSI VevoStrain endocardial (b) and epicardial (a) radial strain showing the same value.

agreement by Bland Altman plot that indicated greater variability (Figure 3), which is in concordance with previous reports $[2,11,12,21,22]$.

Lack of reproducibility has been reported as a major drawback of 2D STE, especially in RS analysis [12, 21-23]. However, there was good reproducibility in our interobserver test for variability in the measurements of LS, CS, and RS on both systems. Nevertheless, the exact reason for greater variability experienced in RS analysis is uncertain and was not apparent from our study. It is presumed that inadequate tracking of the epicardial border and respiratory-related lung artefacts (especially in the antero/lateral segments) play a major contributing role [22].

\section{Limitations}

LV borders were traced semiautomatically by both systems during strain analysis; the two systems are however designed to analyze different cardiac muscle layers. EchoPAC analyzes midmyocardium while VevoStrain analyzes endocardium and epicardium that may inadvertently introduce variability in comparison. Furthermore, it is noted that, without flexibility to adjust individual point, the ROI width remains fixed around the whole circumference in EchoPAC; thus overestimation or underestimation was likely to have occurred during strain analysis. Lastly, our current observations were derived from healthy mice; it will be interesting to ascertain if similar differences between the two systems are replicated in diseased animal models. Nevertheless, similar changes in radial strain recorded with Vivid 7 ([13]; as reported in Figure 2) and Vevo 2100 ([14]; as reported in Table 3) ultrasound systems were separately observed in C57/B6 mice with LV dysfunction by 2 independent groups previously.

\section{Conclusions}

Our study showed that there is a good agreement in LS in the 3 myocardial layers between Vevo 2100 and Vivid 7 ultrasound systems which lends credence to validity of findings from experimental models. Our strain analysis showed that, similar to healthy human subjects [19], there is a gradient of contractile deformation from endocardium towards epicardium in mouse model that may be useful for detailing early changes in myocardial performance in a muscle layer-centric and cardiac segment-specific manner for monitoring disease progression in pathophysiological conditions.

\section{Conflict of Interests}

There is no conflict of interests to declare.

\section{Acknowledgments}

This study was supported by grants from the National Research Foundation Singapore (NRF2008-CRP003-02), the Goh Foundation (Duke-NUS GCR/2013/0008 and GCR/ 2013/011), and Biomedical Research Council Singapore (BMRC 13/1/96/686).

\section{References}

[1] V. Mor-Avi, R. M. Lang, L. P. Badano et al., "Current and evolving echocardiographic techniques for the quantitative evaluation of cardiac mechanics: ASE/EAE consensus statement on methodology and indications endorsed by the Japanese society of echocardiography," European Journal of Echocardiography, vol. 12, no. 3, pp. 167-205, 2011. 
[2] T. H. Marwick, "Consistency of myocardial deformation imaging between vendors," European Journal of Echocardiography, vol. 11, no. 5, pp. 414-416, 2010.

[3] A. Fontana, A. Zambon, F. Cesana, C. Giannattasio, and G. Trocino, "Tissue doppler, triplane echocardiography, and speckle tracking echocardiography: different ways of measuring longitudinal myocardial velocity and deformation parameters. A comparative clinical study," Echocardiography, vol. 29, no. 4, pp. 428-437, 2012.

[4] L. H. Frank, Q. Yu, R. Francis et al., "Ventricular rotation is independent of cardiac looping: a study in mice with situs inversus totalis using speckle-tracking echocardiograph," Journal of the American Society of Echocardiography, vol. 23, no. 3, pp. 315$323,2010$.

[5] V. Chetboul, "Advanced techniques in echocardiography in small animals," Veterinary Clinics of North America: Small Animal Practice, vol. 40, no. 4, pp. 529-543, 2010.

[6] Y. Li, C. D. Garson, Y. Xu et al., "Quantification and MRI validation of regional contractile dysfunction in mice post myocardial infarction using high resolution ultrasound," Ultrasound in Medicine and Biology, vol. 33, no. 6, pp. 894-904, 2007.

[7] A. Bhan, A. Sirker, J. Zhang et al., "High-frequency speckle tracking echocardiography in the assessment of left ventricular function and remodeling after murine myocardial infarction," The American Journal of Physiology-Heart and Circulatory Physiology, vol. 306, no. 9, pp. H1371-H1383, 2014.

[8] J. Korinek, J. Wang, P. P. Sengupta et al., "Two-dimensional strain-a Doppler-independent ultrasound method for quantitation of regional deformation: validation in vitro and in vivo," Journal of the American Society of Echocardiography, vol. 18, no. 12, pp. 1247-1253, 2005.

[9] B. H. Amundsen, T. Helle-Valle, T. Edvardsen et al., "Noninvasive myocardial strain measurement by speckle tracking echocardiography: validation against sonomicrometry and tagged magnetic resonance imaging," Journal of the American College of Cardiology, vol. 47, no. 4, pp. 789-793, 2006.

[10] O. Gjesdal, T. Helle-Valle, E. Hopp et al., "Noninvasive separation of large, medium, and small myocardial infarcts in survivors of reperfused ST-elevation myocardial infarction: a comprehensive tissue Doppler and speckle-tracking echocardiography study," Circulation: Cardiovascular Imaging, vol. 1, no. 3, pp. 189-196, 2008.

[11] A. Manovel, D. Dawson, B. Smith, and P. Nihoyannopoulos, "Assessment of left ventricular function by different speckletracking software," European Journal of Echocardiography, vol. 11, no. 5, pp. 417-421, 2010.

[12] P. Biaggi, S. Carasso, P. Garceau et al., "Comparison of two different speckle tracking software systems: does the method matter?" Echocardiography, vol. 28, no. 5, pp. 539-547, 2011.

[13] Y. Peng, Z. B. Popović, N. Sopko et al., "Speckle tracking echocardiography in the assessment of mouse models of cardiac dysfunction," The American Journal of Physiology-Heart and Circulatory Physiology, vol. 297, no. 2, pp. H811-H820, 2009.

[14] M. Bauer, S. Cheng, M. Jain et al., "Echocardiographic speckletracking based strain imaging for rapid cardiovascular phenotyping in mice," Circulation Research, vol. 108, no. 8, pp. 908916, 2011.

[15] A. S. Otto and T. Edvardsen, "Tissue doppler and speckle tracking echocardiography," in The Practice of Clinical Echocardiography, C. M. Otto and S. Kaplan, Eds., pp. 115-137, Elsevier, New York, NY, USA, 2007.
[16] S. K. Hanneman, "Design, analysis, and interpretation of method-comparison studies," AACN Advanced Critical Care, vol. 19, no. 2, pp. 223-234, 2008.

[17] E. Mavrides, D. Holden, J. M. Bland, A. Tekay, and B. Thilaganathan, "Intraobserver and interobserver variability of transabdominal Doppler velocimetry measurements of the fetal ductus venosus between 10 and 14 weeks of gestation," Ultrasound in Obstetrics and Gynecology, vol. 17, no. 4, pp. 306310, 2001.

[18] R. Ram, D. M. Mickelsen, C. Theodoropoulos, and B. C. Blaxall, "New approaches in small animal echocardiography: imaging the sounds of silence," The American Journal of PhysiologyHeart and Circulatory Physiology, vol. 301, no. 5, pp. H1765H1780, 2011.

[19] M. Leitman, M. Lysiansky, P. Lysyansky et al., "Circumferential and longitudinal strain in 3 myocardial layers in normal subjects and in patients with regional left ventricular dysfunction," Journal of the American Society of Echocardiography, vol. 23, no. 1, pp. 64-70, 2010.

[20] U. Adamu, F. Schmitz, M. Becker, M. Kelm, and R. Hoffmann, "Advanced speckle tracking echocardiography allowing a threemyocardial layer-specific analysis of deformation parameters," European Journal of Echocardiography, vol. 10, no. 2, pp. 303308, 2009.

[21] E. Gayat, H. Ahmad, L. Weinert, R. M. Lang, and V. Mor-Avi, "Reproducibility and inter-vendor variability of left ventricular deformation measurements by three-dimensional speckletracking echocardiography," Journal of the American Society of Echocardiography, vol. 24, no. 8, pp. 878-885, 2011.

[22] L. P. Koopman, C. Slorach, C. Manlhiot et al., "Assessment of myocardial deformation in children using digital imaging and communications in medicine (DICOM) data and vendor independent speckle tracking software," Journal of the American Society of Echocardiography, vol. 24, no. 1, pp. 37-44, 2011.

[23] L. P. Koopman, C. Slorach, W. Hui et al., "Comparison between different speckle tracking and color tissue doppler techniques to measure global and regional myocardial deformation in children," Journal of the American Society of Echocardiography, vol. 23, no. 9, pp. 919-928, 2010. 


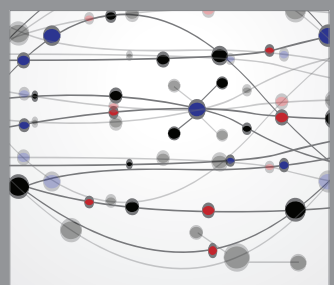

The Scientific World Journal
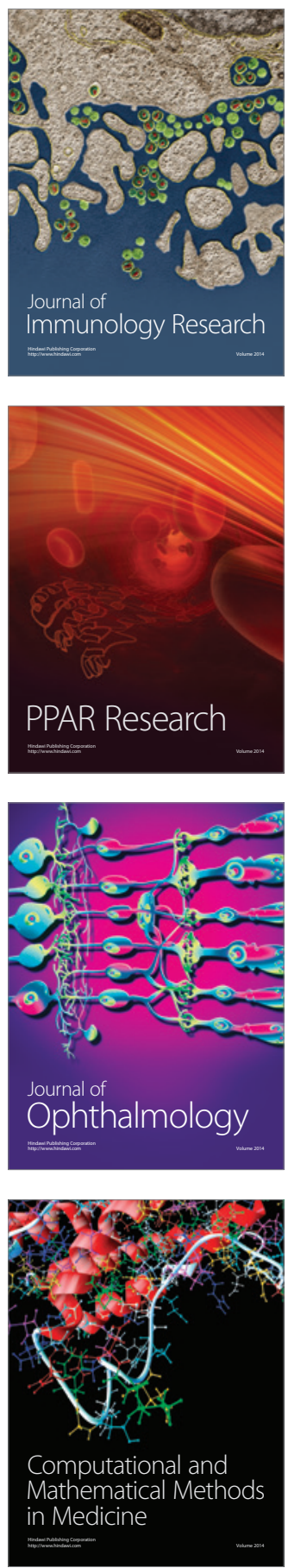

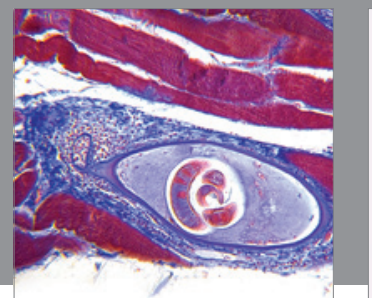

Gastroenterology

Research and Practice
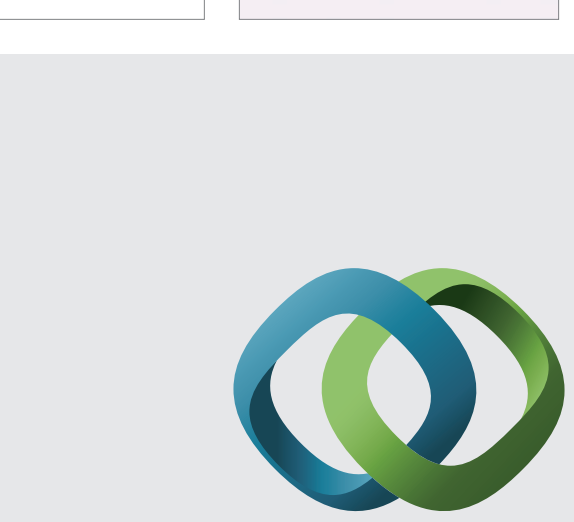

\section{Hindawi}

Submit your manuscripts at

http://www.hindawi.com
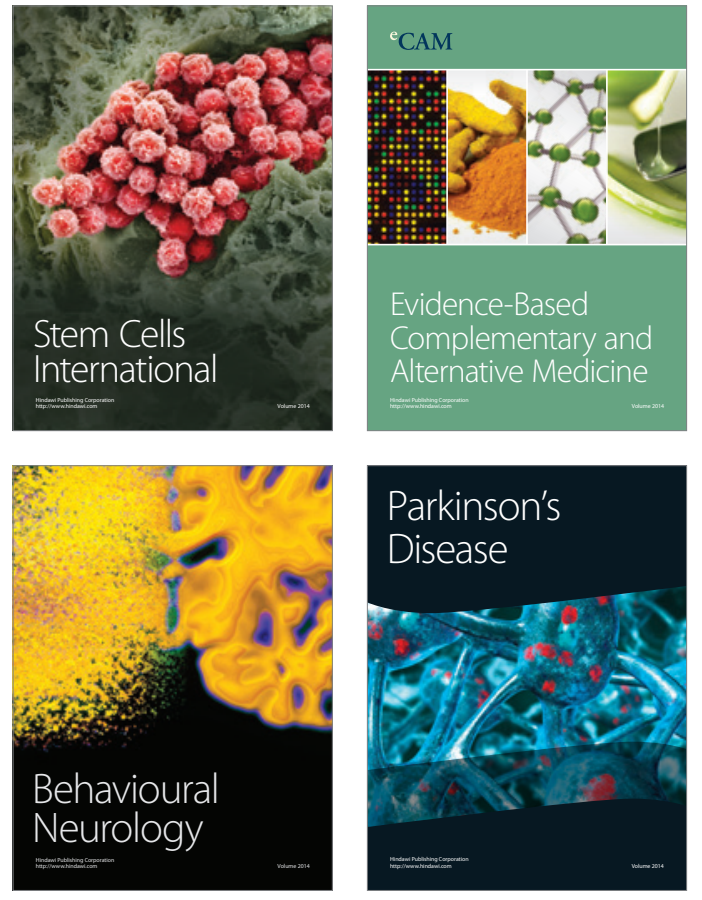
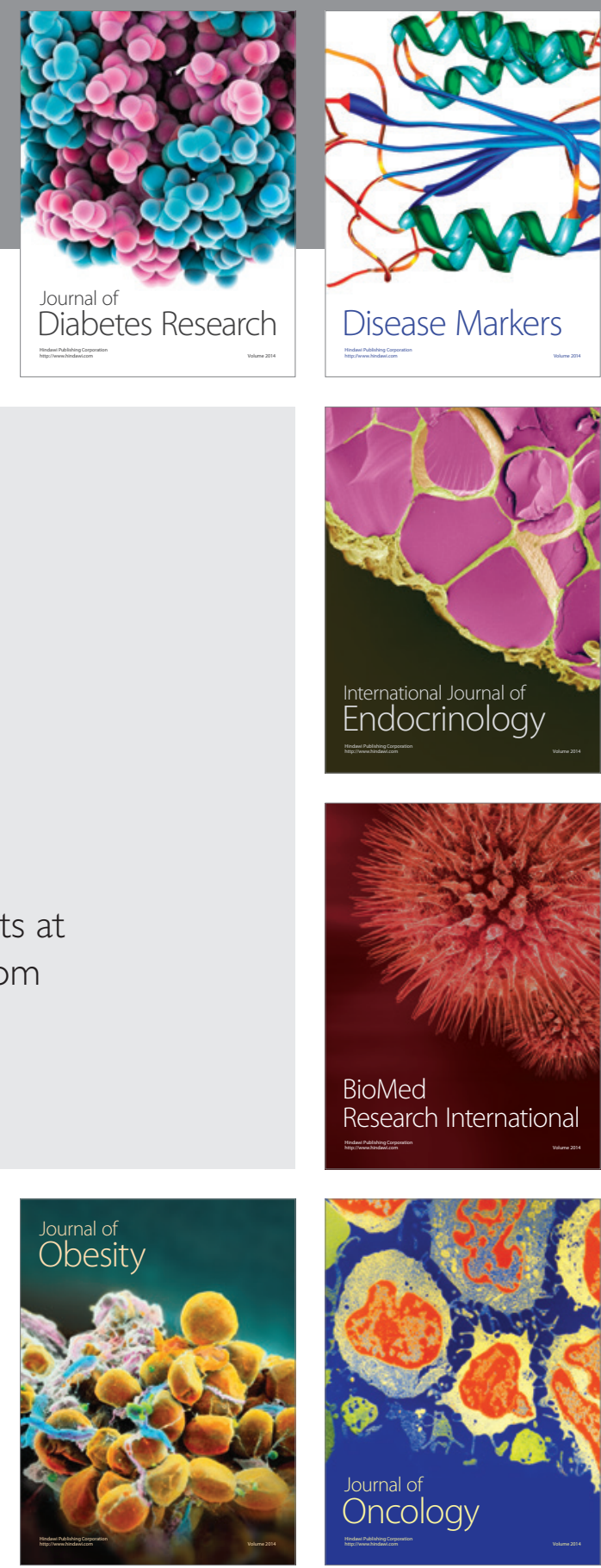

Disease Markers
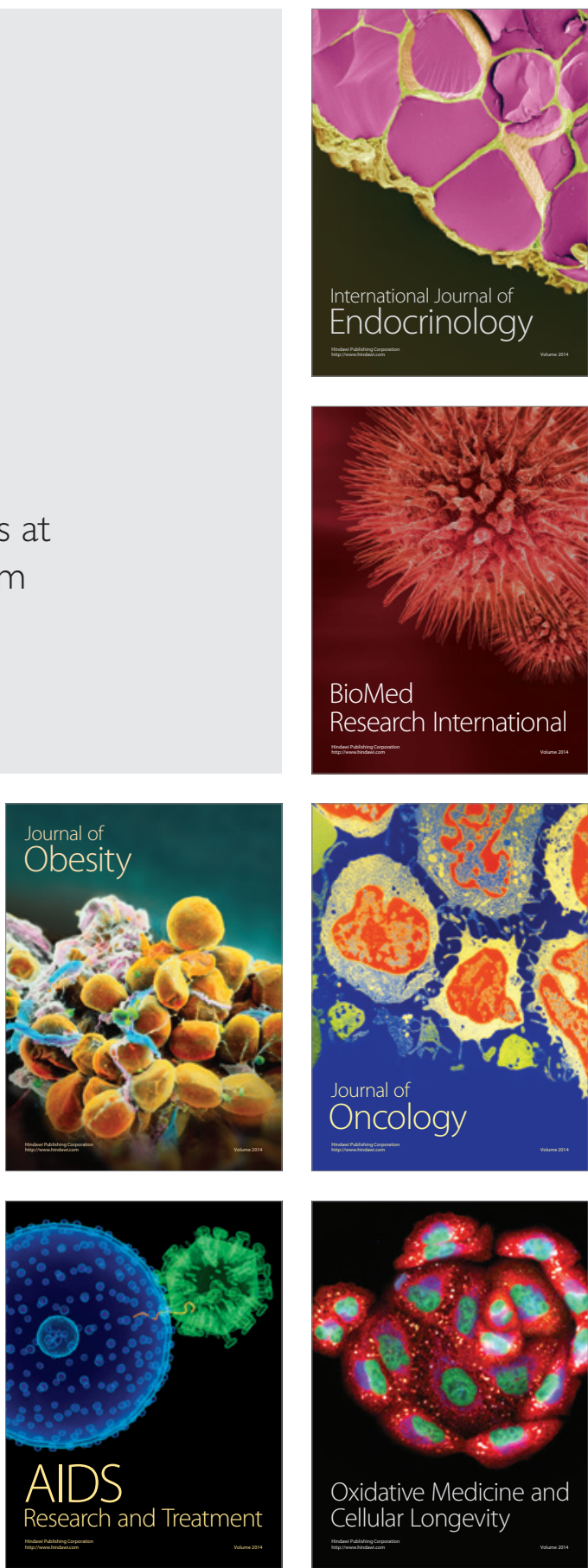